\title{
Energy of harmonic functions and Gromov's proof of Stallings' theorem
}

\author{
M. Kapovich
}

October 29, 2018

\begin{abstract}
We provide the details for Gromov's proof of Stallings' theorem on groups with infinitely many ends using harmonic functions. The main technical result of the paper is a compactness theorem for a certain family of harmonic functions.
\end{abstract}

\section{Introduction}

In his essay [3, Pages 228-230], Gromov gave a proof of the Stallings' theorem on groups with infinitely many ends using harmonic functions. The goal of this paper is to provide the details for Gromov's arguments. The main bulk of the paper is devoted to the proof of a compactness theorem for a certain family of harmonic functions. The corresponding statements are contained in Steps 2 and 4 of Gromov's argument. The rest of our proof closely follows Gromov's.

Let $M$ be a complete Riemannian manifold of bounded geometry, which has infinitely many ends. Suppose that there exists a number $R$ such that every point in $M$ belongs to an $R$-neck, i.e., an $R$-ball which separates $M$ into at least three unbounded components. (This property is immediate if $M$ admits a cocompact isometric group action.)

Let $\bar{M}:=M \cup \operatorname{Ends}(M)$ denote the compactification of $M$ by its space of ends. Given a continuous function $\chi: \operatorname{Ends}(M) \rightarrow\{0,1\}$, let

$$
h=h_{\chi}: \bar{M} \rightarrow[0,1]
$$

denote the continuous extension of $\chi$, so that $h \mid M$ is harmonic. Let $H(M)$ denote the space of harmonic functions

$$
\left\{h=h_{\chi}, \chi: \operatorname{Ends}(M) \rightarrow\{0,1\} \text { is nonconstant }\right\} .
$$

We give $H(M)$ the topology of uniform convergence on compacts in $M$. Let $E$ : $H(M) \rightarrow \mathbb{R}_{+}=[0, \infty)$ denote the energy functional.

Definition 1.1. Given the manifold $M$, define its energy gap $e(M)$ as

$$
e(M):=\inf \{E(h): h \in H(M)\} .
$$


If $M$ admits an isometric group action $G \curvearrowright M$, then $G$ acts on $H(M)$ preserving the functional $E$. Therefore $E$ projects to a lower semi-continuous (see Lemma 4.3) functional $E: H(M) / G \rightarrow \mathbb{R}_{+}$, where we give $H(M) / G$ the quotient topology. Our main objective is to prove

Theorem 1.2. 1. $e(M) \geq \mu>0$, where $\mu$ depends only on $R, \lambda_{1}(M)$ and geometry of $M$.

2. If $M$ admits a cocompact isometric group action, then $E: H(M) / G \rightarrow \mathbb{R}_{+}$is proper in the sense that

$$
E^{-1}([0, T])
$$

is compact for every $T \in \mathbb{R}_{+}$. In particular, $e(M)$ is attained.

Sketch of the proof. With every harmonic function $h=h_{\chi} \in H(M)$ we associate a finite set $K_{I} \subset M$ of the centers $x$ of type 1 special $R$-necks $N(x)$. Roughly speaking, these necks encode the partition of $\operatorname{Ends}(M)$ into the subsets $\chi^{-1}(0), \chi^{-1}(1)$. For all but one component $L$ of $M \backslash N(x), \chi \mid \operatorname{Ends}(L)$ is constant. We also verify that the function $\chi$ is constant on $\operatorname{Ends}\left(M^{\prime}\right)$ for each component $M^{\prime}$ of

$$
M \backslash \bigcup_{x \in K_{I}} \operatorname{int}(N(x))
$$

Every neck $N(x)$ centered at $x \in K_{I}$, "contributes" at least $\mu>0$ to the energy of $h$. This establishes the inequality

$$
e(M) \geq \mu>0
$$

If $E(h) \leq E$, we also obtain an upper bound on the cardinality of $K_{I}$ : $\left|K_{I}\right| \leq \kappa_{1}(E)$.

Suppose that $h_{n}=h_{\chi_{n}} \in H(M)$ is a sequence of functions with $E\left(h_{n}\right) \leq E$. The corresponding sets $K^{(n)}=K_{I}\left(\chi_{n}\right)$ break into subsets $K_{i}^{(n)}$ of uniformly bounded diameter, so that the distance between distinct subsets diverges to infinity as $n \rightarrow \infty$. Using the group $G$, we normalize the functions $h_{n}$ so that $K_{1}^{(n)}$ is contained in a fixed compact subset of $M$. Then the sequence $\left(h_{n}\right)$ subconverges to a harmonic function $h: M \rightarrow[0,1]$. Since each neck $N(x), x \in K_{1}^{(n)}$, contributes at least $\mu$ to the energy of each function $h_{n}$, we conclude that $E(h) \geq \mu>0$. Lastly, we need to check that $h$ extends to a function $\chi: \operatorname{Ends}(M) \rightarrow\{0,1\}$ (a priori, this extension might attain other values in $[0,1]$ as well). This follows from the "uniform connectedness" considerations and uniform estimates for the behavior of the functions $h_{n}$ at the points far away from $K^{(n)}$.

Although it is not needed for the group-theoretic applications, we will also prove

Theorem 1.3. (Finiteness theorem.) Suppose that $M$ admits a cocompact isometric group action. Then $H(M)$ contains only finitely many $G$-orbits of functions $h \in$ $H(M)$ for which $E(h)<e(M)+\mu / 2$.

Acknowledgements. This paper was motivated by numerous discussions with Mohan Ramachandran, to whom I am grateful for many valuable references and suggestions. During the writing of this paper the I was partially supported by the NSF grants DMS-04-05180 and DMS-05-54349. This paper was written when I was visiting Max Plank Institute for Mathematics in Sciences in Leipzig. 


\section{Preliminaries}

Throughout this paper, we let $M$ be a complete Riemannian manifold of bounded geometry, i.e., its injectivity radius is bounded from below by some $C_{1}>0$ and the absolute value of the sectional curvature is bounded from above by some $C_{2}<\infty$. We say that a constant $C$ depends only on geometry of $M$ if it depends only on dimension of $M$, and the numbers $C_{1}$ and $C_{2}$.

Notation 2.1. For a subset $N \subset M$ let $N^{c}$ denote $M \backslash \operatorname{int}(N)$.

Notation 2.2. Given a subset $N \subset M$, let $B_{R}(N)$ denote the collection of points in $M$ which are within distance $\leq R$ from $N$. Thus, $B_{R}(x)$ is the closed $R$-ball centered at $x$.

Notation 2.3. For subsets $S, T \subset M$, define

$$
\operatorname{dist}(S, T):=\inf \{d(x, y): x \in S, y \in T\} .
$$

We will assume that $M$ has infinitely many ends. We say that a metric ball $N=B_{r}(x) \subset M$ is an $r$-neck if $N^{c}$ has at least three unbounded components.

Assumption 2.4. There exist a number $R$ such that $R$-necks cover $M$.

For instance, this assumption holds if $M$ admits a cocompact isometric group action. We fix $R$ satisfying the above assumption from now on and will refer to $R$-necks simply as necks.

Theorem 2.5. Under the above assumption, $M$ is non-amenable, i.e., its Cheeger constant is positive:

$$
\eta(M)=\inf \left\{\frac{\operatorname{Area}(\partial C)}{\operatorname{Vol}(C)}: C \subset M\right\}>0 .
$$

Here the infimum is taken over all compact subsets $C \subset M$ with piecewise-smooth boundary and nonempty interior.

Proof: See [8].

Let $\lambda_{1}(M)$ denote the 1 -st eigenvalue of $M$. Then, by Cheeger's theorem (see [9, Page 91]), we have

$$
\lambda_{1}(M) \geq \eta^{2}(M) / 4
$$

In particular,

$$
\lambda_{1}(M)>0
$$

Theorem 2.6. Let $M$ be a Riemannian manifold of bounded geometry, so that $\lambda_{1}(M)>0$. Then, every continuous function $\chi: \operatorname{Ends}(M) \rightarrow\{0,1\}$, admits a continuous extension to a (unique) function

$$
h=h_{\chi}: \bar{M} \rightarrow[0,1]
$$

whose restriction to $M$ is harmonic. 
Proof: This theorem was proven by Kaimanovich and Woess in [5, Theorem 5] using probabilistic methods (they also proved it for functions $\chi$ with values in $[0,1]$ ). In the the context of Kähler manifolds, the theorem was proven in [7, Theorem 2.6]. In Section 5, we will present a proof of this theorem provided by Mohan Ramachandran.

Suppose that $\chi_{1}, \chi_{2}: \operatorname{Ends}(M) \rightarrow\{0,1\}$ are such that

$$
\chi_{1} \leq \chi_{2}
$$

Then, by the maximum principle,

$$
h_{\chi_{1}} \leq h_{\chi_{2}}
$$

If the equality is attained at some point of $M$, then $\chi_{1}=\chi_{2}$.

We now restrict to continuous functions $\chi: \operatorname{Ends}(M) \rightarrow\{0,1\}$.

Lemma 2.7. Each function $h=h_{\chi}$ has finite energy

$$
E(h)=\int_{M}|\nabla h|^{2}
$$

Proof: The assertion follows immediately from Lemma 5.3 (i) [9, Page 71].

A subset of $\operatorname{Ends}(M)$ is called clopen if it is both open and closed.

Definition 2.8 (Clusters). A clopen subset of $\chi^{-1}(i)$ is called an $i$-cluster with respect to the function $\chi$. When $i$ is irrelevant, we refer to an $i$-cluster as a cluster.

A domain in $M$ is a connected properly embedded codimension 0 submanifold $M^{\prime} \subset M$, which has smooth compact boundary. Then

$$
\operatorname{Ends}\left(M^{\prime}\right) \subset \operatorname{Ends}(M)
$$

and

$$
\overline{M^{\prime}} \subset \bar{M}
$$

are continuous embeddings.

Definition 2.9. A domain $M^{\prime} \subset M$ cobounds an $i$-cluster (with respect to the function $\chi)$ if Ends $\left(M^{\prime}\right)$ is a cluster.

\section{Uniform connectivity}

Fix $R>0$ and let $\mathcal{N} \subset M$ be a $\delta$-separated net $(\delta>0)$. In case when $M$ admits a cocompact isometric action of a discrete group $G$, we assume that $\mathcal{N}$ is $G$-invariant. Pick a subset $K \subset \mathcal{N}$ of diameter $\leq r$ and consider its $R$-neighborhood $N=B_{R}(K)$ in $M$. Define $\Phi(K, r)$ as follows. For each component $C$ of $N^{c}$, consider the induced path-metric on $C$. Then let $\Phi(K, r)$ be the maximum (over all $C$ 's) of the diameters of $C \cap N$ with respect to this metric. In other words, $\Phi(K, r)$ equals

$$
\max _{C} \sup \left\{x, y \in C \cap N: \inf _{p \in \Pi_{x y}} \operatorname{length}(p)\right\}
$$


where the $\Pi_{x y}$ is the set of all paths in $C$ connecting $x$ to $y$.

We define the uniform connectivity function

$$
\phi(r):=\sup \{\Phi(K, r): K \subset \mathcal{N}, \operatorname{diam}(K) \leq r\} .
$$

Then $\phi$ is an increasing function. The following lemma is clear:

Lemma 3.1. Suppose that $M$ admits an isometric cocompact group action preserving $\mathcal{N}$. Then $\phi(r)$ is finite for each $r \in \mathbb{R}$.

In general, $\phi(r)$ need not be finite.

Example 3.2. Let $R=1$. Start with the complex plane $\mathbb{C}$ with its flat metric. Let $S_{n}$ denote the double of

$$
\mathbb{C} \backslash\left(B_{1}(n) \cup B_{1}(-n)\right)
$$

across its boundary. Smooth out this metric along the boundary of $B_{1}(n) \cup B_{1}(-n)$ to make it Riemannian. Then $\phi(4) \geq n$ for $S_{n}$.

Lastly, take the connected sum of the surfaces $S_{n}(n \geq 3)$ as follows: Remove from each $S_{n}$ one copy of the disk $D_{n}=B_{1}(0)$ and glue $S_{n}$ to $S_{n+1}$ along the boundaries of $D_{n}, D_{n+1}$. Smooth out the resulting metric. This infinite connected sum has infinite $\phi(4)$.

Assumption 3.3. From now on we assume that $M$ is such that $\phi(r)$ is finite for each $r \in \mathbb{R}$.

One can easily see that finiteness of $\phi$ is independent of the choice of the net $\mathcal{N}$, number $R$ and is invariant under quasi-isometries. (We do not need these properties.)

Let $K^{(n)}=\left\{x_{n, 1}, \ldots, x_{k, n}\right\}$ denote a sequence of subsets of cardinality $\leq k$ in $M$. Since $[0, \infty]^{k^{2}}$ is compact, after passing to a subsequence, we can assume that for each $i, j$, there is a limit

$$
\lim _{n} d\left(x_{n, i}, x_{n, j}\right) \in[0, \infty]
$$

Thus, we obtain

Lemma 3.4. After passing to a subsequence in the sequence $\left(K^{(n)}\right)$, we can break $K^{(n)}$ as the disjoint union of nonempty subsets

$$
K^{(n)}=\bigcup_{i=1}^{l} K_{i}^{(n)}
$$

so that

1. $\operatorname{diam}\left(K_{i}^{(n)}\right) \leq D<\infty$, for all $i=1, \ldots, l, n \in \mathbb{N}$.

2. $\lim _{n \rightarrow \infty} \operatorname{dist}\left(K_{i}^{(n)}, K_{j}^{(n)}\right)=\infty$ for $i \neq j$.

When $n$ is sufficiently large, we obtain that for all $i \neq j$,

$$
\operatorname{dist}\left(B_{R}\left(K_{i}^{(n)}\right), B_{R}\left(K_{j}^{(n)}\right)\right)>d:=\max _{m} \phi\left(\operatorname{diam}\left(K_{m}^{(n)}\right)\right) .
$$

We now take one of the sets $K=K^{(n)}$ and its partition

$$
K^{(n)}=\bigcup_{i=1}^{l} K_{i}^{(n)}
$$


as in the above lemma. By abusing the notation, we will abbreviate $K_{i}^{(n)}$ as $K_{i}$, $i=1, . ., l$.

Consider the covering of $M$ by the sets $B_{R}\left(K_{1}\right), \ldots, B_{R}\left(K_{l}\right)$ and by the connected components $C_{1}, \ldots, C_{m}$ of $B_{R}(K)^{c}$. Then the nerve of this covering is a finite graph $\Gamma$ without loop and bigons. We will use the notation $K_{i}, C_{j}$ for the vertices of this graph corresponding to the sets $K_{i}, C_{j}$.

We will say that $\Gamma$ is dual to $K$.

Lemma 3.5. The graph $\Gamma$ is a tree provided that (1) holds. In other words, whenever $x, y \in M$ are disconnected by $\operatorname{int}\left(B_{R}(K)\right)$, there exists $i$ so that $x, y$ are disconnected by $\operatorname{int}\left(B_{R}\left(K_{i}\right)\right)$.

Proof: Suppose that $\Gamma$ is not a tree. Then it contains a shortest cycle which we denote

$$
K_{1}-C_{1}-K_{2}-\ldots-C_{s}-K_{1}
$$

Let $x \in C_{1} \cap B_{R}\left(K_{1}\right), y \in C_{s} \cap B_{R}\left(K_{1}\right)$. Then $x$ and $y$ belong to the same connected component of $M \backslash \operatorname{int}\left(B_{R}\left(K_{1}\right)\right)$. Therefore, by the inequality (1), there exists a path $p$ in $M \backslash \operatorname{int}\left(B_{R}\left(K_{1}\right)\right)$ disjoint from

$$
B_{R}\left(K \backslash K_{1}\right)
$$

connecting $x$ and $y$. See Figure 1. Therefore this path has to be contained in both $C_{1}$ and $C_{s}$. Hence, $C_{1}=C_{s}$. Contradiction.

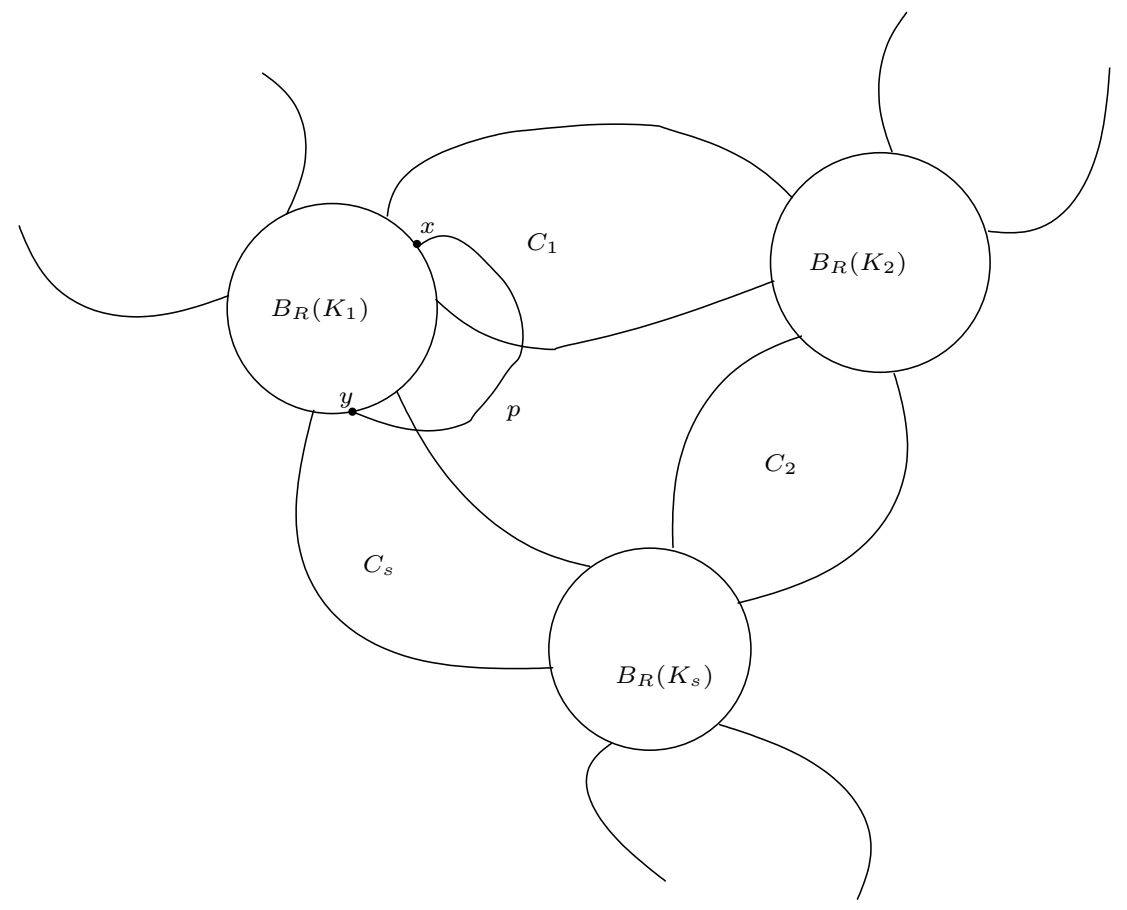

Figure 1: 


\section{Estimates on harmonic functions on $M$}

Gradient estimate, see [9, Page 17]. There exists a constant $C=C_{\text {grad }}$ which depends only on geometry of $M$, so that for every positive harmonic function $u$ : $M \rightarrow \mathbb{R}$ we have

$$
|\nabla u(x)| \leq C u(x)
$$

for all $x \in M$.

\section{Decay estimates for harmonic functions.}

Proposition 4.1. There exists a function $\rho(\epsilon, D, k), \epsilon>0, D>0$, which depends only on the geometry of $M$, so that the following holds.

Let $M^{\prime} \subset M$ be a domain whose boundary $\partial M$ is the union of at most $k$ subsets $\partial_{i} M^{\prime}$, each of diameter $\leq D$. Set $r:=\rho(\epsilon, D, k)$. Let $h: M \rightarrow(0,1)$ be a harmonic function which vanishes on Ends $\left(M^{\prime}\right)$. Then:

For every $x \in T:=M^{\prime} \backslash B_{r}\left(\partial M^{\prime}\right)$, we have

$$
h(x) \leq \epsilon .
$$

Proof: Given the fact that $\lambda_{1}(M)>0$, the proof follows by repeating the arguments of Lemma 5.3 (part (iii)) in [9, Chapter II]. (This lemma establishes uniform exponential decay for harmonic functions which converge to zero at infinity.) See also [6, Lemmata $1.1,1.2]$.

Corollary 4.2. Suppose that $M_{0}, M_{1} \subset M$ are noncompact disjoint domains, so that $\operatorname{diam}\left(\partial M_{i}\right) \leq D$, and $\chi \mid \operatorname{Ends}\left(M_{i}\right) \equiv i, i=0,1$. Let $\gamma$ denote a shortest geodesic segment connecting $\partial M_{0}$ to $\partial M_{1}$ and let length $(\gamma) \leq l$. Then

$$
E\left(h \mid B_{1}\left(\gamma \cup M_{0} \cup M_{1}\right)\right) \geq \mu(l, D),
$$

where the function $\mu(l, D)>0$ depends only on the geometry of $M$.

Proof: Take $\epsilon=1 / 10$. By applying Proposition 4.1 to the functions $h \mid M_{0}$ and $(1-h) \mid M_{1}$, we find points $x_{i} \in M_{i}$ such that $d\left(x_{i}, \partial M_{i}\right)=\rho=\rho(\epsilon, D, 1)$ and

$$
h\left(x_{0}\right) \leq \epsilon, \quad h\left(x_{1}\right) \geq 1-\epsilon .
$$

It follows that $d\left(x_{0}, x_{1}\right) \leq 2 \rho+l$ and

$$
\left|h\left(x_{0}\right)-h\left(x_{1}\right)\right| \geq 1-2 \epsilon .
$$

Let $y_{i} \in \partial M_{i}^{\prime}$ denote the end-points of $\gamma$. Connect $y_{i}$ to $x_{i}$ by the shortest geodesic segments $\alpha_{i}, i=0,1$. Let $\beta:=\alpha_{0} \cup \gamma \cup \alpha_{1}$. Then length of $\beta$ is at most $2 \rho+l$.

By the mean value theorem, there exists a point $y \in \beta$ so that

$$
|\nabla h(y)| \geq \frac{1-2 \epsilon}{2 \rho+l} .
$$

Therefore

$$
E\left(h \mid B_{1}(y)\right) \geq \mu(l, D)=\text { Const } \frac{0.64}{(2 \rho+l)^{2}},
$$

where Const depends only on geometry of $M$. 
Lemma 4.3. The energy function $E: H(M) \rightarrow \mathbb{R}_{+}$is lower semi-continuous.

Proof: Let $h=h_{\chi} \in H(M)$ be the limit

$$
h=\lim _{n \rightarrow \infty} h_{n}, \quad h_{n} \in H(M) .
$$

Let $\epsilon>0$. Pick a sufficiently large ball $B_{r}(o) \subset M$, so that each unbounded component $M_{i}(i=1, \ldots, q)$ of $B_{r}(o)^{c}$ cobounds a cluster with respect to $\chi$. Then, since $E(h)$ is finite (Lemma 2.7), there exists $\rho \geq r$, so that for each $i$,

$$
E\left(h \mid M_{i} \backslash B_{\rho}(o)\right) \leq \epsilon .
$$

Let $C$ denote the compact in $M$ which is the union of $B_{\rho}(o)$ and the compact components of $B_{r}(o)^{c}$. As uniform convergence $h_{n} \mid C$ implies uniform convergence of these functions in $C^{1}$-norm (by the gradient estimate), we obtain

$$
E(h \mid C)=\lim _{n \rightarrow \infty} E\left(h_{n} \mid C\right) .
$$

Therefore,

$$
E(h) \leq E(h \mid C)+q \epsilon \leq \lim i n f_{n \rightarrow \infty} E\left(h_{n}\right) .
$$

Since $q$ is constant and $\epsilon$ is arbitrarily small, we obtain

$$
E(h) \leq \lim i n f_{n \rightarrow \infty} E\left(h_{n}\right) .
$$

\section{An existence theorem for harmonic functions}

Theorem 5.1. Let $\chi: \operatorname{Ends}(M) \rightarrow\{0,1\}$ be a continuous function. Then $\chi$ admits a harmonic extension to $M$.

Proof: (M. Ramachandran.) Let $\varphi$ denote a smooth extension of $\chi$ to $M$ so that $d \varphi$ is compactly supported.

We let $W_{o}^{1,2}(M)$ denote the closure of $C_{c}^{\infty}(M)$ with respect to the norm

$$
\|u\|:=\|u\|_{L_{2}}+\sqrt{E(u)} .
$$

Consider the affine subspace of functions

$$
\mathcal{F}:=\varphi+W_{o}^{1,2}(M) \subset L_{l o c}^{2}(M) .
$$

Then the energy is well-defined on $\mathcal{F}$ and we set $E:=\inf _{f \in \mathcal{F}} E(f)$.

Note that, since $\mathcal{F}$ is affine, for $u, v \in \mathcal{F}$ we also have

$$
\frac{u+v}{2} \in \mathcal{F},
$$

in particular,

$$
E\left(\frac{u+v}{2}\right) \geq E
$$

and we set

$$
E(u, v):=2 E\left(\frac{u+v}{2}\right)-\frac{E(u)+E(v)}{2} .
$$


The latter equals

$$
E(u, v):=\int_{M}\langle\nabla u, \nabla v\rangle
$$

in the case when $u, v$ are smooth. We thus obtain

$$
E(u, v) \geq 2 E-\frac{E(u)+E(v)}{2}
$$

for all $u, v \in \mathcal{F}$. Hence,

$$
E(u-v)=E(u)+E(v)-2 E(u, v) \leq 2 E(u)+2 E(v)-4 E .
$$

Pick a sequence $u_{n} \in \mathcal{F}$ such that

$$
\lim _{n \rightarrow \infty} E\left(u_{n}\right)=E .
$$

Then, according to (2),

$$
E\left(u_{m}-u_{m}\right) \leq 2 E\left(u_{n}\right)+2 E\left(u_{m}\right)-4 E=2\left(E\left(u_{n}\right)-E\right)+2\left(E\left(u_{m}\right)-E\right) .
$$

Since $\lambda:=\lambda_{1}(M)>0$, we obtain

$$
\lambda \int_{M} f^{2} \leq E(f)
$$

for all $f \in W_{o}^{1,2}(M)$. Therefore, the functions $v_{n}:=u_{n}-\varphi \in W_{o}^{1,2}(M)$ satisfy

$$
\left\|v_{n}-v_{m}\right\| \leq\left(2+\lambda^{-1}\right)\left(E\left(u_{n}\right)-E+E\left(u_{m}\right)-E\right) .
$$

Hence, the sequence $\left(v_{n}\right)$ is Cauchy in $W_{o}^{1,2}(M)$. Set

$$
v:=\lim _{n} v_{n}, u:=\varphi+v \in \mathcal{F} .
$$

By semicontinuity of energy, $E(u)=E$. Therefore, $u$ is harmonic and, hence, smooth. Since $d \varphi$ is compactly supported, the function $v$ is also harmonic away from a compact subset $K \subset M$. By the inequality (3), we have

$$
\int_{M} v^{2} \leq \lambda^{-1} E(v)<\infty
$$

Let $r>0$ denote the injectivity radius of $M$. Pick a base-point $o \in M$. Then (4) implies that there exists a function $\rho: M \rightarrow \mathbb{R}_{+}$which converges to 0 as $d(x, o) \rightarrow \infty$, so that

$$
\int_{B_{r}(x)} v^{2}(x) \leq \rho(x)
$$

for all $x \in M$. By the gradient estimate, there exists $C_{1}<\infty$ so that

$$
\sup _{B_{r}(x)} v^{2} \leq C_{1} \inf _{B_{r}(x)} v^{2}
$$

provided that $d(x, K) \geq r$. Therefore,

$$
v^{2}(x) \leq \frac{C_{1}}{\operatorname{Vol}\left(B_{r}(x)\right)} \int_{B_{r}(x)} v^{2} \leq C_{2} \rho(x) .
$$

Thus

$$
\lim _{d(x, o) \rightarrow \infty} v(x)=0 .
$$

Therefore the harmonic function $u$ extends to the function $\chi$ on $\operatorname{Ends}(M)$. 


\section{Geometry of necks}

Let $R$ be as in Section 2. Pick $\mathcal{N} \subset M$, a $\delta$-separated $R$-net in $M$. If $M$ admits an isometric cocompact action $G \curvearrowright M$, we assume that this net is $G$-invariant. For $x \in \mathcal{N}$ we let $N(x):=B_{R}(x)$ denote the corresponding neck.

Definition 6.1. Given a nonconstant function $\chi:$ Ends $(M) \rightarrow\{0,1\}$, we say that a neck $N=N(x)$ is a regular $\theta-$ neck $(\theta \in\{0,1\})$ if for all but one components $M^{\prime}$ of $N^{c}$ satisfy

$$
\chi \mid \operatorname{Ends}\left(M^{\prime}\right) \equiv \theta \text {. }
$$

A neck which is not regular, is called special.

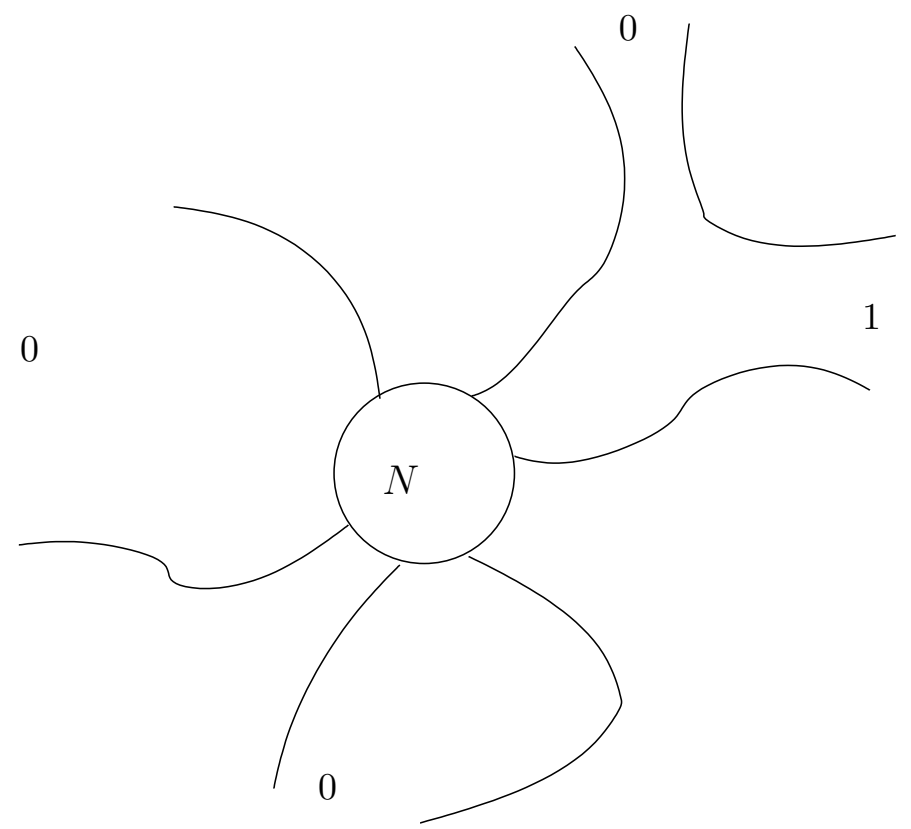

Figure 2: Regular neck.

There are two types of special necks:

Type 1. There exists at most one (unbounded) component $M^{\prime}$ of $N^{c}$ which does not cobound a cluster and there are at least two (unbounded) components $M_{0}, M_{1}$ of $N^{c}$ so that $\chi \mid \operatorname{Ends}\left(M_{i}\right) \equiv i, i=0,1$.

Type 2. There are at least who components $M_{1}, M_{2}$ of $M \backslash \operatorname{int}(N)$ which do not cobound clusters.

Let $K \subset \mathcal{N}$ denote the set of centers of special necks and let $K_{I}$ and $K_{I I}$ denote the subsets of $K$ consisting of the centers of type 1 and 2 necks respectively.

Remark 6.2. Suppose that there exists a special neck $N(x)$ of type 1, so that each component of $N(x)^{c}$ cobounds a cluster. Then every neck $N(y)$ disjoint from $N(x)$ is regular.

Lemma 6.3. Suppose that $N_{i}=N\left(x_{i}\right)$ are regular $\theta_{i}$-necks, $i=1,2$, which have nonempty intersection. Then $\theta_{1}=\theta_{2}$. 


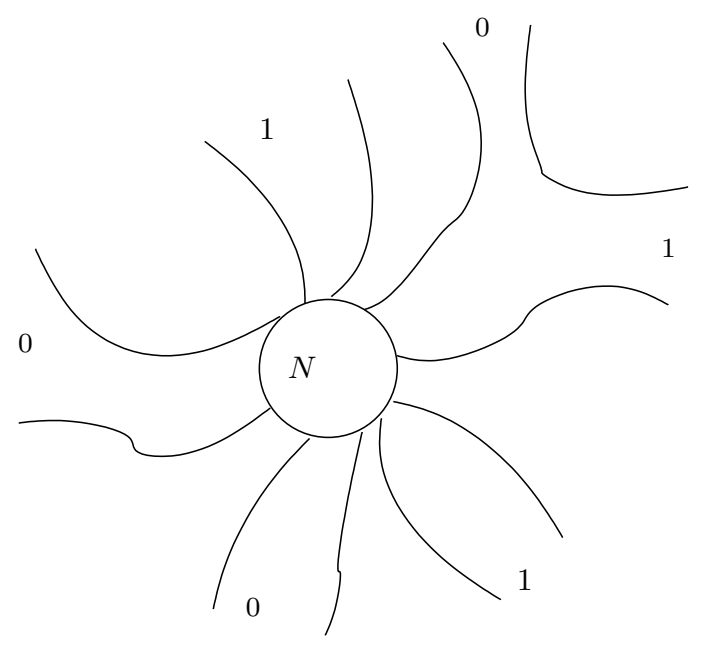

Type 1 special neck

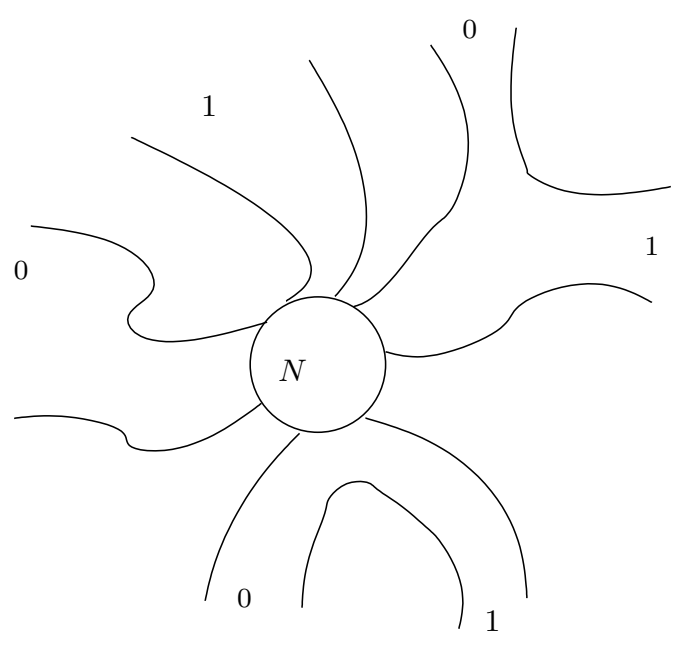

Type 2 special neck

Figure 3: Special necks.

Proof: We will consider the most interesting case, when both $N_{i}^{c}$ contain exactly one complementary component $M_{i}^{\prime}$ which does not cobound a cluster and will leave the remaining cases to the reader.

Then

$$
M_{2}^{\prime} \cup\left(N_{2} \backslash N_{1}\right) \subset M_{1}^{\prime} .
$$

Suppose that $\theta_{1} \neq \theta_{2}$. Let $M_{2} \subset N_{2}^{c}$ be an (unbounded) component. If $M_{2}$ is not contained in $M_{1}^{\prime}$, then it is contained in a component $C$ of $N_{2}^{c}$ so that $\chi \mid \operatorname{Ends}(C) \equiv \theta_{2}$. Therefore $\chi \mid \operatorname{Ends}(C) \equiv \theta_{2}$ which contradicts our assumption that $\theta_{1} \neq \theta_{2}$.

Hence, we have

$$
M_{2} \subset M_{1}^{\prime}
$$

Similarly, every component $M_{1} \subset N_{1}^{c}$ is contained in $M_{2}^{\prime}$. But this implies that all unbounded components of $N_{1}^{c}$ are contained in $M_{1}^{\prime}$. Therefore $N_{1}^{c}$ has only one unbounded component, i.e., $M_{1}^{\prime}$. Contradiction.

Lemma 6.4. Suppose that $x_{i}$ is sufficiently close (in the topology of $\bar{M}$ ) to a point $\xi \in \chi^{-1}(\theta) \subset \operatorname{Ends}(M)$. Then the neck $N\left(x_{i}\right)$ is a regular $\theta$-neck.

Proof: Pick a base-point $o \in M$. Let $U \subset \bar{M}=M \cup$ Ends $(M)$ be an open neighborhood of $\xi$ so that $\chi \mid U \equiv \theta$. Then (by the definition of topology on $\bar{M}$ ) there exists $r_{0}$ such that for all $r \geq r_{0}$, if $C$ is a component of $M \backslash B_{r}(o)$ which intersects $U$, then $\operatorname{Ends}(C) \subset U$.

Hence, there exists a neighborhood $V \subset U \subset \bar{M}$ of $\xi$, so that for each $x \in V \cap M$, one of the unbounded complementary components $C$ of $B_{R}(x)$ will contain $B_{r_{0}}(o)$ and the other unbounded components $C^{\prime}$ will be such that $\operatorname{Ends}\left(C^{\prime}\right) \subset U$. Therefore, $\chi \mid \operatorname{Ends}\left(C^{\prime}\right) \equiv \theta$. It follows that $B_{R}(x)$ is a regular $\theta$-neck.

Corollary 6.5. If $\chi$ is nonconstant, then there exists at least one special neck in $M$. 
Proof: Since $\chi$ is nonconstant, the above lemma implies that $M$ contains at least one regular $i$-neck for $i=0,1$. Now the assertion follows Lemma 6.3 and connectedness of $M$.

Corollary 6.6. The subset $K \subset \mathcal{N}$ of centers of special necks $N(x)$ is finite.

Proof: The statement follows from compactness of $\bar{M}$ combined with Lemma 6.4,

Lemma 6.7. Let $N=N(x)$ be a type 2 special neck. Then for every component $M^{\prime}$ of $N^{c}$ which does not bound a cluster, $M^{\prime} \cup N$ contains a type 1 special neck $N(y)$.

Proof: Let $K_{I I}^{\prime}$ denote the subset of $K_{I I}$ consisting of points $y \in \mathcal{N}$ such that $N(y) \subset$ $M^{\prime} \cup N$. Let $k\left(M^{\prime}\right)$ denote the cardinality of $K_{I I}^{\prime}$. We prove Lemma using induction on $k\left(M^{\prime}\right)$.

1. Suppose $k\left(M^{\prime}\right)=1$, i.e., $K_{2}^{\prime}$ consists only of $v$. If $M^{\prime} \cup N$ contains no special necks besides $N(x)$, then $M^{\prime}$ cobounds a cluster. This is a contradiction. Thus, $M^{\prime} \cup N$ contains a type 2 special neck.

2. Suppose the assertion holds whenever $k\left(M^{\prime}\right) \leq k$. Consider $M^{\prime}$ with $k\left(M^{\prime}\right)=$ $k+1$. Let $z \in K_{2}^{\prime}$; then the neck $N(z)$ is special of type 2. At least one of the unbounded components $M^{\prime \prime}$ of $N(z)^{c}$ (which does not cobound a cluster in $M$ ) is contained in $M^{\prime}$ and is disjoint from $N(x)$. Then $k\left(M^{\prime \prime}\right) \leq k$. Therefore, by the induction assumption, $M^{\prime \prime} \cup N(z) \subset M^{\prime} \cup N(x)$ contains a type 1 special neck $N(y)$.

Lemma 6.8. Every unbounded component of $B_{R}\left(K_{I}\right)^{c}$ cobounds a cluster.

Proof: If not, then there exists a component $M^{\prime} \subset B_{R}\left(K_{I}\right)^{c}$ which contains a type 2 special neck $N(x)$, whose non-cluster complementary component $M^{\prime \prime}$ is entirely contained in $M^{\prime}$. Therefore, according to Lemma 6.7, $M^{\prime \prime}$ contains a type 1 special neck $N(y)$. However $y \notin K_{I}$. Contradiction.

\section{Compactness theorem}

The goal of this section is to prove Theorem 1.2, Let $\chi: \operatorname{Ends}(M) \rightarrow\{0,1\}$ be a nonconstant continuous function. Let $K=K(\chi) \subset \mathcal{N}$ be as in the previous section. Define $\mu=\mu_{M}:=\mu(2 R, R)>0$, where $\mu(\cdot, \cdot)$ is the function defined in Corollary 4.2.

Lemma 7.1. For $x \in K_{I}$, let $M_{i}(x)$ denote components of $N(x)^{c}$ which cobound $i$-clusters, $i=0,1$. Then

$$
E\left(h \mid M_{0}(x) \cup M_{1}(x) \cup N(x)\right) \geq \mu .
$$

Proof: The assertion immediately follows from Corollary 4.2 .

Corollary 7.2. If $M^{\prime}$ is a component of $N(x)^{c}$ which does not cobound a cluster, then

$$
E\left(h_{\chi} \mid M^{\prime} \cup N(x)\right) \geq \mu .
$$


Proof: Since $M^{\prime}$ does not cobound a cluster, there exists a special neck $N(y)$ contained in $M^{\prime \prime}:=M^{\prime} \cup N$. If this special neck is of type 1, we are done by Lemma 7.1. If $N(y)$ is of type 2, then, by Lemma 6.7, $M^{\prime \prime}$ contains a special neck of type 1. Hence, we are again done by Lemma 7.1 .

Corollary 7.3. For every $h \in H(M)$, we have

$$
E(h) \geq \mu .
$$

Thus, $e(M) \geq \mu>0$.

Lemma 7.4. There exists a function $\kappa_{1}(E)$ (which depends only on geometry of $M$ ) such that if $E\left(h_{\chi}\right) \leq E$, then the cardinality of $K_{I}$ is at most $\kappa_{1}(E)$.

Proof: For $x \in K_{I}$ let $M_{i}(x)$ denote components of $N(x)^{c}$ which cobound $i$-clusters, $i=0,1$. It is clear that if $N(x) \cap N(y)=\emptyset$, then the four sets

$$
M_{i}(x), M_{i}(y), i=0,1
$$

are pairwise disjoint. It follows from Lemma 7.1, that

$$
E\left(h \mid M_{0}(x) \cup M_{1}(x) \cup N(x)\right) \geq \mu=\mu(2 R, R)
$$

for every $x \in K_{I}$. Thus, the cardinality of $K_{I}$ is at most $E / \mu$.

One can also bound the number of type 2 necks as well, provided that $E\left(h_{\chi}\right)$ is sufficiently small:

Proposition 7.5. Suppose that $E(h)<E=e(M)+\mu / 2$. There exists a function $\kappa_{2}(E)$ such that the cardinality of $K_{I I}$ is at most $\kappa_{2}(E)$.

We do not need this fact and leave it without a proof. The Proposition follows from the proof of Finiteness Theorem, see $\sqrt[8]{8}$. Observe, however, that if $E(h)$ is large comparing to $e(M)$, then one cannot have a uniform upper bound on the cardinality of $K_{I I}$.

We are now ready to prove properness of the function $E: H(M) / G \rightarrow \mathbb{R}_{+}$, assuming that $G$ is a discrete subgroup of $\operatorname{Isom}(M)$ which acts cocompactly on $M$.

Suppose that $h_{n}=h_{\chi_{n}} \in H(M)$ is a sequence of harmonic functions with uniformly bounded energy $E\left(h_{n}\right) \leq E<\infty$. For each $n$ we define the set

$$
K^{(n)}=K_{I}\left(\chi_{n}\right) \subset \mathcal{N}
$$

of centers of special necks of type 1. By Lemma 7.4, the cardinality of each $K^{(n)}$ is at most $k \leq \kappa_{1}(E)$. We break each $K^{(n)}$ as the union

$$
K^{(n)}=\sqcup_{i=1}^{l} K_{i}^{(n)}
$$

as in Lemma 3.4, so that

$$
\operatorname{diam}\left(K_{i}^{(n)}\right) \leq D, \quad \forall n, \forall i,
$$

and

$$
\lim _{n} \operatorname{dist}\left(K_{i}^{(n)}, K_{j}^{(n)}\right)=\infty
$$


for $i \neq j$. Let $\Gamma=\Gamma_{n}$ denote the dual graph for the above partition of $K^{(n)}$. Since the number of vertices and edges of $\Gamma$ is uniformly bounded, after passing to a subsequence we can assume that $\Gamma$ does not depend on $n$.

By applying elements of $G$ and passing to a subsequence, we can assume that a certain point $x_{1 n} \in K_{1}^{(n)}$ is a point $o \in \mathcal{N}$ which does not depend on $n$. Therefore, without loss of generality, we may assume that $K_{1}^{(n)}$ does not depend on $n$ either.

Let $M_{n, 1}, \ldots, M_{n, s}$ denote the unbounded components of $B_{R}\left(K^{(n)}\right)^{c}$ which are adjacent to $B_{R}\left(K_{1}^{(n)}\right)$. Let $M_{1}^{\prime}, \ldots, M_{t}^{\prime}$ be the unbounded components of $B_{R}\left(K_{1}^{(n)}\right)^{c}$ which are adjacent to $B_{R}\left(K_{1}^{(n)}\right)$. Because $\Gamma$ is a tree, it follows that $s=t$ and that distinct components $M_{n, i}$ lie in distinct components $M_{i}^{\prime}$ for every sufficiently large $n$, and all $i=1, \ldots, t$. See Figure 4.

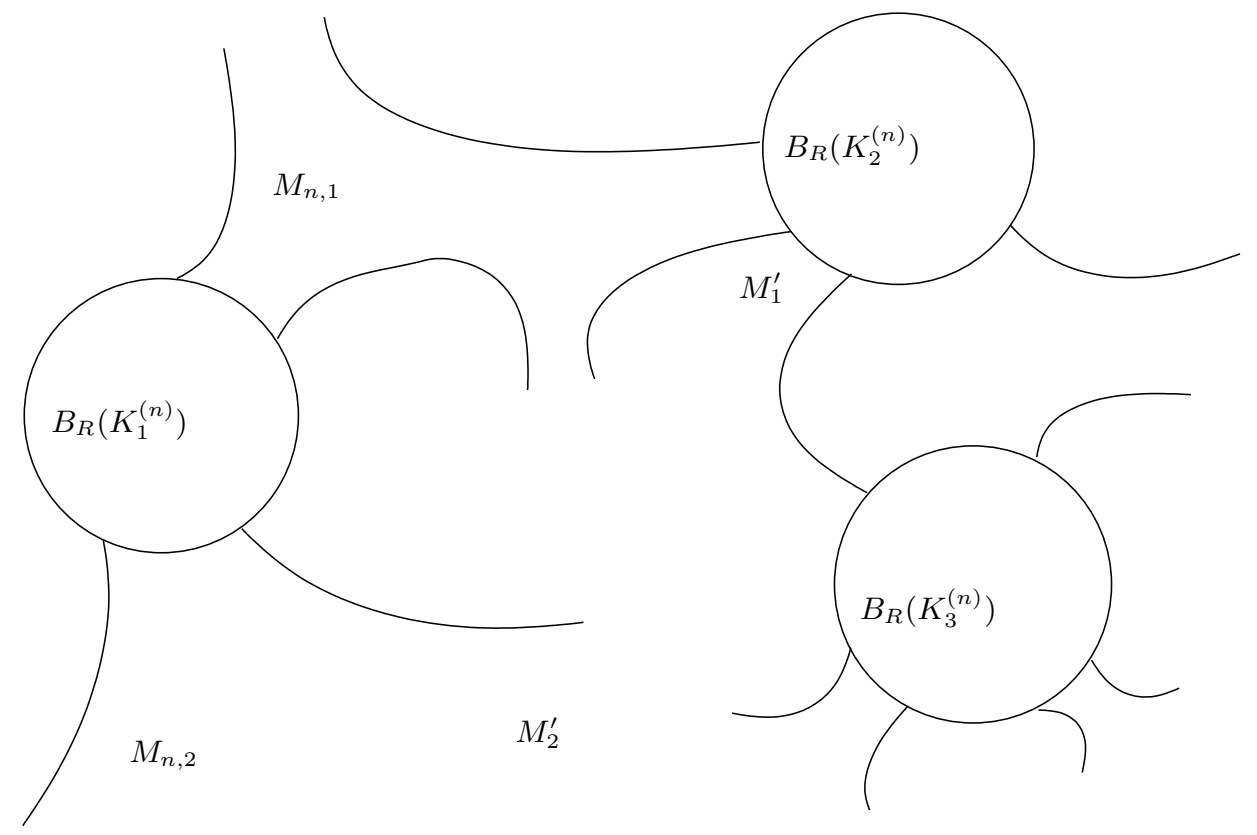

Figure 4:

Recall that each $M_{n, i}$ cobounds a cluster in $\operatorname{Ends}(M)$ (with respect to $\chi_{n}$ ). Let $\theta_{i}$ denote the constant value of $\chi_{n}$ on $\operatorname{Ends}\left(M_{n, i}\right)$. (After passing to a subsequence, we may assume that these constants are independent of $n$.) Note that, since $K_{1}^{(n)}$ is the set of centers of type 1 special neck, there are $i, j$ so that $\theta_{i} \neq \theta_{j}$.

Since the functions $h_{n}$ take values in $(0,1)$, by the gradient estimate, the family $\left(h_{n}\right)$ is equicontinuous. Therefore, there exists a limit $h:=\lim _{n} h_{n}$, which is again a harmonic function.

Lemma 7.6. For each $i=1, \ldots, s$,

$$
\lim _{d(x, o) \rightarrow \infty} h(x)=\theta_{i}
$$

where $x \in M_{i}^{\prime}$.

Proof: Let $\epsilon>0$. Pick $x \in M_{i}^{\prime} \backslash B_{\rho(\epsilon, D, k)}\left(\partial M_{i}^{\prime}\right)$. Then, for sufficiently large $n \geq n_{0}$,

$$
x \in B_{R}\left(K^{(n)}\right)^{c} .
$$


Therefore, by Proposition 4.1, for $n \geq n_{0}$,

$$
\left|h_{n}(x)-\theta_{i}\right| \leq \epsilon
$$

Hence, the function $h$ extends to a continuous function $\chi: \operatorname{Ends}(M) \rightarrow\{0,1\}$ :

$$
\chi \mid \operatorname{Ends}\left(M_{i}^{\prime}\right) \equiv \theta_{i}
$$

Since $\theta_{i} \neq \theta_{j}$ for some $i, j$, we obtain that $h \in H(M)$. Since $E$ is lower semicontinuous, the energy functional $E: H(M) / G \rightarrow \mathbb{R}_{+}$is proper. It is now clear that $E$ attains the minimum $e(M)>0$. This concludes the proof of Theorem 1.2 ,

\section{Finiteness theorem}

In this section we prove Finiteness Theorem 1.3. Suppose that there are infinitely many $G$-cosets of functions $h_{n} \in H(M)$ with $E(h)<e(M)+\mu / 2$. Then, after passing to a subsequence, and using the notation of the previous section, $\operatorname{diam}\left(K^{(n)}\right) \rightarrow \infty$ and $h=\lim _{n} h_{n}$. As before, we normalize the functions $h_{n}$ using the group $G$ and pass to a subsequence, so that

$$
K^{(n)}=\bigcup_{i=1}^{l} K_{i}^{(n)}
$$

where $K_{1}^{(n)}$ is independent of $n$. Let $h=\lim _{n} h_{n}$. Pick $\epsilon>0$, so that $\epsilon<\mu / 4$. As in the proof of Theorem 1.2, we get a sufficiently large compact subset $C \subset M$ so that for all $n \geq n_{0}$ we have

$$
\begin{gathered}
E(h \mid M \backslash C) \leq \epsilon, \quad E\left(h_{n} \mid M \backslash C\right) \leq \epsilon, \\
\left|E(h \mid C)-E\left(h_{n} \mid C\right)\right| \leq \epsilon .
\end{gathered}
$$

On the other hand, (for large $n) M \backslash C$ contains at least one type 1 special neck $N:=N\left(x_{n}\right), x_{n} \in K^{(n)} \backslash K_{1}^{(n)}$. Let $M_{i}\left(x_{n}\right), i=0,1$ denote the components of $N^{c}$ which cobound $i$-clusters with respect to $\chi_{n}$. Then

$$
U_{n}:=N \cup M_{0}\left(x_{n}\right) \cup M_{1}\left(x_{n}\right)
$$

is disjoint from the compact $C$. According to Lemma 7.1 ,

$$
E\left(h_{n} \mid U_{n}\right) \geq \mu
$$

Putting these inequalities together, we obtain

$$
E(h) \leq E(h \mid C)+\epsilon \leq E\left(h_{n}\right)+2 \epsilon-\mu<e(M),
$$

since $E\left(h_{n}\right)<e(M)+\mu / 2$. However, $h \in H(M)$ and $e(M)=\min \{E(h), h \in H(M)\}$. Contradiction. 


\section{Proof of Stallings' theorem}

The goal of this section is to present the rest of Gromov's proof of the Stallings' theorem on groups with infinitely many ends. The following was proven by Stallings [10] for torsion-free groups, his proof was extended by Bergman [2] to groups with torsion:

Theorem 9.1 (Stallings, Bergman). Let $G$ be a finitely-generated group with infinitely many ends. Then $G$ splits nontrivially as a graph of groups with finite edge groups.

Proof: Our argument is a slightly expanded version of Gromov's proof in [3, Pages 228-230]. Since $G$ is finitely-generated, it admits a cocompact isometric properly discontinuous action $G \curvearrowright M$ on a connected Riemannian manifold $M$. For instance, if $G$ is $k$-generated, and $F$ is a Riemann surface of genus $k$, we have an epimorphism

$$
\phi: \pi_{1}(F) \rightarrow G
$$

Then $G$ acts isometrically and cocompactly on the covering space $M$ of $F$ so that $\pi_{1}(M)=\operatorname{ker}(\phi)$. Thus, $M$ has infinitely many ends. The manifold $M$ has bounded geometry since it covers a compact Riemannian manifold.

Let $H(M)$ denote the space of harmonic functions $h: M \rightarrow(0,1)$ as in the Introduction. According to Theorem 1.2, there exists a function $h \in H(M)$ with minimal energy $E(h)=e(M)>0$. Then, for every $g \in G$, the function

$$
g^{*} h:=h \circ g
$$

has the same energy as $h$ and equals

$$
h_{g^{*}(\chi)}
$$

For $g \in G$, define

$$
g_{+}(h):=\max \left(h, g^{*}(h)\right), \quad g_{-}(h):=\min \left(h, g^{*}(h)\right) .
$$

Set

$$
\Lambda:=\left\{x: h(x)=g^{*} h(x)\right\}=\{x: h(x)=h(g(x))\} \subset M .
$$

Lemma 9.2.

$$
E\left(g_{+}(h)\right)+E\left(g_{-}(h)\right)=2 E(h) .
$$

Proof: Without loss of generality, we may assume that $h \neq g^{*}(h)$. Then the set $\Lambda$ has measure zero (see e.g. [4] or [1]). Set

$$
M_{-}:=\left\{x \in M: h(x)>g^{*} h(x)\right\}, M_{+}:=\left\{x \in M: h(x)<g^{*} h(x)\right\} .
$$

We obtain:

$$
\begin{gathered}
E\left(g_{+}(h)\right)+E\left(g_{-}(h)\right)= \\
\int_{M_{-}}|\nabla h(x)|^{2}+\int_{M_{+}}\left|\nabla g^{*} h(x)\right|^{2}+\int_{M_{-}}\left|\nabla g^{*} h(x)\right|^{2}+\int_{M_{+}}|\nabla h(x)|^{2}=
\end{gathered}
$$




$$
=E(h)+E\left(g^{*}(h)\right)=2 E(h) .
$$

Note that the functions $g_{+}(h), g_{-}(h)$ have continuous extension to $\bar{M}$ (since $h$ does and $G$ acts on $\bar{M}$ by homeomorphisms). By construction, the restrictions

$$
\chi_{+}:=g_{+}(h)\left|\operatorname{Ends}(M), \quad \chi_{-}:=g_{-}(h)\right| \operatorname{Ends}(M)
$$

take the values 0 and 1 on $\operatorname{Ends}(M)$. Let

$$
h_{ \pm}:=h_{\chi_{ \pm}}
$$

denote the corresponding harmonic functions on $M$. Then

$$
\begin{gathered}
E\left(h_{ \pm}\right) \leq E\left(g_{ \pm}(h)\right), \\
E\left(h_{+}\right)+E\left(h_{-}\right) \leq 2 E(h)=2 e(M) .
\end{gathered}
$$

Note that it is, a priori, possible that $\chi_{-}$or $\chi_{+}$is constant. Set

$$
G_{c}:=\left\{g \in G: \chi_{-} \text {or } \chi_{+} \text {is constant }\right\} .
$$

We first analyze the set $G \backslash G_{c}$. For $g \notin G_{c}$, both $h_{-}$and $h_{+}$belong to $H(M)$ and, hence,

$$
E\left(h_{+}\right)=E\left(h_{-}\right)=E(h)=e(M) .
$$

Therefore,

$$
E\left(g_{+}(h)\right)=E\left(h_{+}\right), \quad E\left(g_{-}(h)\right)=E\left(h_{-}\right) .
$$

It follows that $g_{ \pm}(h)$ are both harmonic. Since

$$
g_{-}(h) \leq g_{+}(h),
$$

the maximum principle implies that either $g_{-}(h)=g_{+}(h)$ or $g_{-}(h)<g_{+}(h)$. Hence, the set $\Lambda$ is either empty or equals the entire $M$, in which case $g^{*}(h)=h$. Therefore, for every $g \in G \backslash G_{c}$ on of the following holds:

1. $g^{*} h=h$.

2. $g^{*} h(x)<h(x), \forall x \in M$.

3. $g^{*} h(x)>h(x), \forall x \in M$.

Thus, the set

$$
L:=h^{-1}\left(\frac{1}{2}\right)
$$

is precisely-invariant under the elements of $G \backslash G_{c}$ : for every $g \in G \backslash G_{c}$, either

$$
g(L)=L
$$

or

$$
g(L) \cap L=\emptyset .
$$

We now consider the elements of $G_{c}$. Suppose that $g$ is such that $\chi_{-}=0$. Then

$$
g^{*}(\chi) \leq 1-\chi
$$

and, hence,

$$
g^{*}(h) \leq 1-h .
$$


Since these functions are harmonic, in the case of the equality at some $x \in M$, by the maximum principle we obtain $g^{*}(h)=1-h$. The latter implies that

$$
g(L)=L
$$

If

$$
g^{*}(h)<1-h
$$

then $g(L) \cap L=\emptyset$. The same argument applies in the case when $\chi_{+}$is constant.

To summarize, for every $g \in G$ one of the following holds:

$$
g^{*} h=h, \quad g^{*} h<h, \quad g^{*} h>h, \quad g^{*} h=1-h, \quad g^{*} h<1-h, \quad g^{*} h>1-h .
$$

We conclude that $L$ is precisely-invariant under the action of the entire group $G$. Moreover, if $g(L)=L$ then either $g^{*} h=h$ or $g^{*} h=1-h$. Since $L$ is compact, its stabilizer $G_{L}$ in $G$ is finite.

By construction, the hypersurface $L$ separates $M$ in at least two unbounded components.

Since $L$ is compact, there exists $t \in(0,1) \backslash \frac{1}{2}$ sufficiently close to $\frac{1}{2}$, which is a regular value of $h$, so that the hypersurface $S:=h^{-1}(t)$ is still precisely-invariant under $G$. Let $G_{S} \subset G_{L}$ denote the stabilizer of $S$ in $G$.

It is now rather standard that $G$ splits nontrivially over a subgroup of $G_{S}$. We present a proof for the sake of completeness. (The proof is straightforward under the assumption that $S$ is connected, but requires extra work in general.) We proceed by constructing a simplicial $G$-tree $T$ on which $T$ acts without inversions, with finite edge-stabilizers and without a global fixed vertex.

Construction of $T$. Consider the family of functions $\mathcal{F}=\left\{f=g^{*} h: g \in G\right\}$. Each function $f \in \mathcal{F}$ defines the wall $W_{f}=\{x: f(x)=t\}$ and the half-spaces $W_{f}^{+}:=\{x: f(x)>t\}, W_{f}^{-}:=\{x: f(x)<t\}$ (these spaces are not necessarily connected).

Let $\mathcal{E}$ denote the set of walls. We say that a wall $W$ separates $x, y \in M$ if

$$
x \in W_{f}^{+}, \quad y \in W_{f}^{-} .
$$

Maximal subsets $V$ of

$$
M^{o}:=M \backslash \bigcup_{f \in \mathcal{F}} W_{f}
$$

consisting of points which cannot be separated from each other by a wall, are called indecomposable subsets of $M^{o}$. Note that such sets need not be connected. Set

$$
\mathcal{V}:=\left\{\text { indecomposable subsets of } M^{o}\right\}
$$

We say that a wall $W$ is adjacent to $V \in \mathcal{V}$ if $W \cap c l(V) \neq \emptyset$.

The next lemma follows immediately from the inequalities (5), provided that $t$ is sufficiently close to $\frac{1}{2}$ :

Lemma 9.3. No wall $W_{f_{1}}$ separates points of another wall $W_{f_{2}}$. 
Lemma 9.4. 1. Let $V \in \mathcal{V}$ and $W \in \mathcal{E}$ be adjacent to $V$. Then, for each component $C$ of $V$, we have $C \cap W \neq \emptyset$.

2. $W \in \mathcal{E}$ is adjacent to $V \in \mathcal{V}$ iff $W \subset \operatorname{cl}(V)$.

Proof: 1. Suppose that $V \subset W^{+}$. A generic point $x \in C$ is connected to $W=W_{f}$ by a gradient curve $p:[0,1] \rightarrow M$ of the function $f$. The curve $p$ crosses each wall at most once. Since $V$ is indecomposable and for sufficiently small $\epsilon>0, p(1-\epsilon) \in V$, it follows that $p$ does not cross any walls. Therefore the image of $p$ is contained in the closure of $C$ and $p(1) \in W \cap \operatorname{cl}(C)$

2. Lemma 9.3 implies that for $x, y \in W^{+}$(resp. $x, y \in W^{-}$) which are sufficiently close to $W$, there is no wall which separates $x$ from $y$. Therefore, such points $x, y$ belong to the same indecomposable set $V^{+}$(resp. $V^{-}$) which is adjacent to $W$ and $W \subset \operatorname{cl}\left(V^{ \pm}\right)$. Clearly, $V^{+}, V^{-}$are the only indecomposable sets which are adjacent to $W$.

Hence, each wall $W$ is adjacent to exactly two elements of $\mathcal{V}$ (contained in $W^{+}, W^{-}$ respectively). We obtain a graph $T$ with the vertex set $\mathcal{V}$ and edge set $\mathcal{E}$, where a vertex $V$ is incident to an edge $W$ iff the wall $W$ is adjacent to the indecomposable set $V$.

From now on, we abbreviate $W_{f_{i}}$ to $W_{i}$.

Lemma 9.5. $T$ is a tree.

Proof: By construction, every point of $M$ belongs to a wall or to an indecomposable set. Hence, connectedness of $T$ follows from connectedness of $M$.

Let

$$
W_{1}-V_{1}-W_{2}-\ldots-W_{k}-V_{k}-W_{1}
$$

be an embedded cycle in $T$. This cycle corresponds to a collection of paths $p_{j}$ : $[0,1] \rightarrow \operatorname{cl}\left(V_{j}\right)$, so that

$$
p_{j}(0) \in W_{j}, \quad p_{j}(1) \in W_{j+1}, j=1, \ldots, k .
$$

The points of $p_{j}([0,1])$ are not separated by any wall, $j=1, \ldots, k$. By Lemma 9.3, the points $p_{j}(1), p_{j+1}(0)$ are not separated by any wall either. Therefore, the points of

$$
\bigcup_{j=1}^{k} p_{j}([0,1])
$$

are not separated by $W_{1}$. However,

$$
p_{1}((0,1]) \subset W_{1}^{+}, \quad p_{k}([0,1)) \subset W_{1}^{-}
$$

or vice-versa. Contradiction.

We next note that $G$ acts naturally on $T$ since the sets $\mathcal{F}, \mathcal{E}$ and $\mathcal{V}$ are $G-$ invariant and $G$ preserves adjacency. If $g\left(W_{f}\right)=W_{f}$, then $g^{*} f=f$, which implies that $g$ preserves $W_{f}^{+}, W_{f}^{-}$. Hence, $g$ fixes the end-points of the edge corresponding to $W$, which means that $G$ acts on $T$ without inversions. The stabilizer of an edge in $T$ corresponding to a wall $W$ is finite, since $W$ is compact and $G$ acts on $M$ properly discontinuously. 
Suppose that $G \curvearrowright T$ has a fixed vertex. This means that the corresponding indecomposable subset $V \subset M$ is $G$-invariant. Since $G$ acts cocompactly on $M$, it follows that $M=B_{r}(V)$ for some $r \in \mathbb{R}_{+}$. The indecomposable subset $V$ is contained in the half-space $W^{+}$for some wall $W$. Since $W$ is compact and $W^{-}$is not, the subset $W^{-}$is not contained in $B_{r}(W)$. Thus $W^{-} \backslash B_{r}(V) \neq \emptyset$. Contradiction.

Therefore $T$ is a nontrivial $G$-tree and we obtain a nontrivial graph of groups decomposition of $G$ where the edge groups are conjugate to subgroups of the finite group $G_{S}$.

\section{References}

[1] C. Bär, On nodal sets for Dirac and Laplace operators, Comm. Math. Phys. 188 (1997), no. 3, 709-721.

[2] G. Bergman, On groups acting on locally finite graphs, Ann. of Math. 88 (1968) 335-340.

[3] M. Gromov, Hyperbolic groups, In: "Essays in group theory", 75-263, Math. Sci. Res. Inst. Publ., 8, Springer, New York, 1987.

[4] R. Hardt and L. Simon, Nodal sets for solutions of elliptic equations, J. Differential Geom. 30 (1989), no. 2, 505-522.

[5] V. Kaimanovich and W. Woess, The Dirichlet problem at infinity for random walks on graphs with a strong isoperimetric inequality, Probab. Theory Related Fields, 91 (1992), no. 3-4, 445-466.

[6] P. Li and J. Wang, Complete manifolds with positive spectrum, J. Differential Geom. 58 (2001), 501-534.

[7] T. Napier and M. Ramachandran, Structure theorems for complete Kähler manifolds and applications to Lefschetz type theorems, Geom. Funct. Anal. 5 (1995), no. 5, 809-851.

[8] C. Pittet, On the isoperimetry of graphs with many ends, Colloq. Math. 78 (1998), N 2, 307-318.

[9] R. Schoen and S.-T. Yau, "Lectures on differential geometry". Conference Proceedings and Lecture Notes in Geometry and Topology, I. International Press, Cambridge, MA, 1994.

[10] J. Stallings, On torsion-free groups with infinitely many ends, Ann. of Math. 88 (1968), 312-334.

Department of Mathematics, 1 Shields Ave., University of California, Davis, CA 95616, USA

kapovich@math.ucdavis.edu 